\title{
Performance Analysis of a Hybrid CDMA/ISMA Protocol for Indoor Wireless Computer Communications
}

\author{
Huub van Roosmalen, Jos Nijhof, and Ramjee Prasad
}

\begin{abstract}
A combination of Direct Sequence Code Division Multiple Access (DS-CDMA) and p-persistent Inhibit Sense Multiple Access (ISMA) protocols is proposed for Indoor Wireless Communications (IWC). The combination of these two protocols is called the hybrid CDMA/ISMA protocol. The performance of the hybrid CDMA/ISMA protocol is analyzed with a Markov model considering Differential Phase Shift Keying (DPSK) modulation. The performance is measured in terms of throughput and delay. The performance is also evaluated using computer simulation of which the results are in close agreement with the Markov model results. The study of indoor wireless communications using a hybrid CDMA/ISMA protocol can be useful for various applications in research and design offices, medical data communication in hospitals, etc.
\end{abstract}

\section{INTRODUCTION}

$\mathbf{T}$ HE performance results of Code Division Multiple Access (CDMA) and Inhibit Sense Multiple Access (ISMA) protocols have been reported separately in numerous papers, e.g., [1]-[8]. In this paper, we propose a hybrid protocol for Indoor Wireless Communications which combines Direct Sequence CDMA with p-persistent ISMA. This protocol has two key features. First of all, it solves the hidden terminal problem by routing all traffic via a central base station. Second, it allows many users with a relatively short transmission code onto the same computer network by having users share transmission codes. The protocol can be used in a wide range of applications where users are semistationary, such as wireless patient monitoring in hospitals or flexible network access for mobile sales persons who check in several times a day with portable computers.

In this paper, we assume perfect power control to avoid the near/far effect. Also, for gaining a full understanding of the protocol, we first consider noiseless nonfading channels and then Rayleigh fading channels with noise. Under these conditions, we investigate the performance of the protocol using two approaches. First, the protocol performance is analyzed using a Markov model, then we verify the model output for small networks by simulation and use the simulation to obtain results for larger networks.

Manuscript received May 26, 1993; revised November 8, 1993. This work was presented at The Fourth International Symposium on Personal, Indoor and Mobile Radio Communications (PIMRC'93), Yokohama, Japan, September 8-11, 1993.

The authors are with the Faculty of Electrical Engineering, Telecommunications and Traffic-Control Systems Group, Delft University of Technology, 2600 GA Delft, The Netherlands.

IEEE Log Number 9215585.

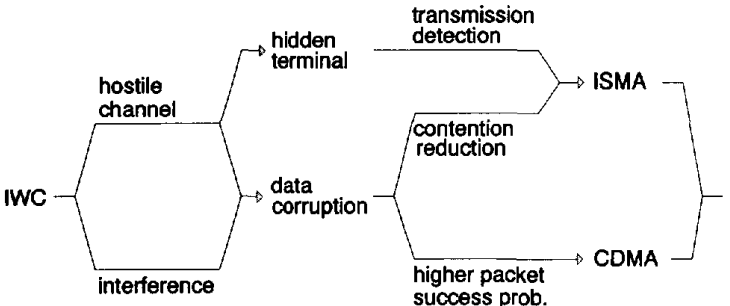

Fig. 1. Combination of CDMA and ISMA protocols for Indoor Wireless Communications.

As it is well known that CDMA improves the survival chance of packets and ISMA limits the contention in the channel, it would be typical to expect that the performance of a hybrid CDMA/ISMA network will be quite good, and at least better than the performance of separate protocols alone (Fig. 1).

For practical systems, implementing full CDMA would mean that the number of codes used equals the number of terminals in the network. In large networks, this can become quite costly in terms of receiver hardware. For each code, a separate receiver is needed. To reduce the number of receivers, we will investigate whether it is feasible to let a number of terminals share the same code. The number of terminals sharing the same code will function using the ISMA protocol. Thus, for a given indoor wireless network, $N_{t}$ users will be divided into $n$ groups with different codes, where each group will have $N_{t} / n$ users with the same code.

This paper is organized as follows. The protocol description is given in Section II. Section III presents the Markov model. The physical model of the system is discussed in Section IV. The bit error probability is derived in Section V. Computational results are discussed in Section VI. Section VII contains the concluding remarks.

\section{PROTOCOL DESCRIPTION}

The network consists of a set of receivers star-connected by wire to a central base station. Around each receiver, we have a cluster of terminals sharing the same transmission code (Fig. 2). When a data packet is generated by the user, the terminal waits for the beginning of the next time slot and transmits the data to its receiver, which forwards it to the base station. The base station then broadcasts the packet to all terminals, and the destination terminal receives the packet. The return channel is not included in our analysis. The base 


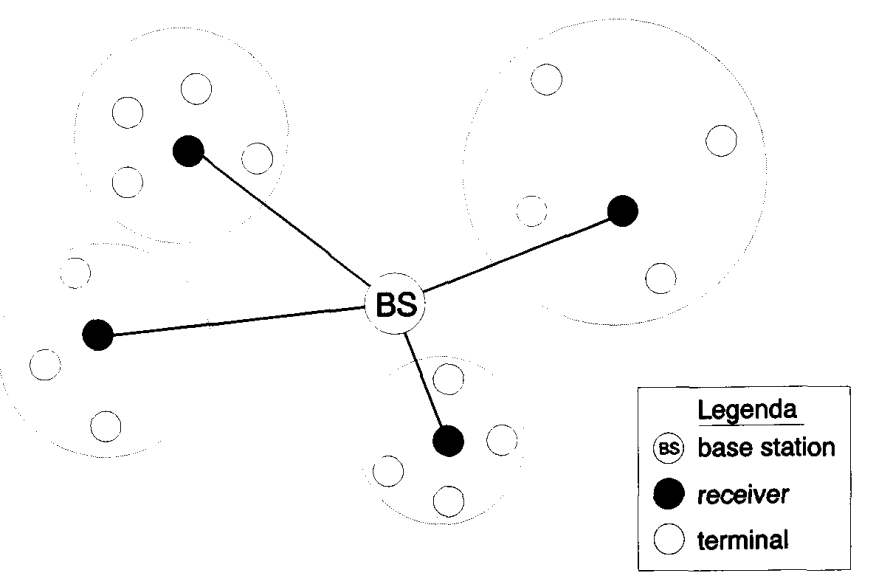

Fig. 2. CDMA/ISMA network architecture.

station is the sole user of the return channel and, hence, there is no contention for the return channel.

The base station controls the flow of traffic with a busy tone which it broadcasts to all terminals. At the beginning of each time slot, the busy tone is interrupted long enough to allow all terminals to start their transmissions. If more than one receiver forwards a packet to the base station, the base station picks a packet at random to service and broadcasts it to all terminals. All other packets are ignored and must be retransmitted in one of the next time slots. Both the receivers and the base station cannot distinguish between an errorless and a damaged packet and process incoming signals regardless. We assume that the terminal being serviced can determine within the same time slot whether the transmission was errorless or not by listening to the broadcast packet.

Terminals in the network have two states: idle or blocked. Initially, all terminals are idle. The arrival of new packets is a Poisson process. Whenever a packet arrives at an idle terminal, the terminal goes into the blocked state and services the packet according to the SDL (Specification and Description Language) diagram in Fig. 3. The terminals have no buffers, so packets arriving at a blocked terminal are dropped.

Each blocked terminal waits for the start of the next time slot before it attempts transmission. Then, a random binary experiment is done to determine whether an actual attempt is made or not, depending on the transmission probability $p_{\mathrm{tr}}$. If the terminal decides to transmit and the transmission was successful, the terminal returns to the idle state, otherwise it repeats the above process. The retransmission delay may be set to zero because the random binary experiment already introduces a delay after a collision.

The physical traffic in the network $T_{p}$ is defined as the sum of the traffic of all terminals flowing across line $A$ in Fig. 3. The throughput $S$ of the network is defined as the fraction of time slots of the return channel carrying successful transmissions, equivalent to the flow of all terminals across $B$ in Fig. 3. The packet delay $D$ is the number of time slots it takes from the arrival of the packet at the terminal until the reception of the last packet bit at the destination.

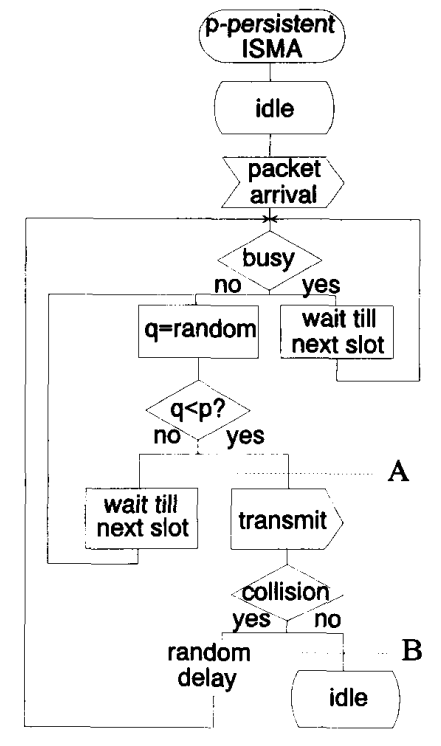

Fig. 3. SDL diagram of terminal transmission protocol.

\section{MARKOV MODEL}

The Markov model is an excellent tool for analyzing network performance because it can be used to predict both transient and steady-state behavior of dynamic processes. The behavior of the network is described by a matrix $\mathbf{P}$ with elements $p_{i, j}$ representing the probability that a system in state $i$ will go to state $j$ in the next time slot. The state vector $\mathbf{v}$ with elements $v_{i}$ contains the probability distribution of finding the system in state $i$. The state distribution of the system in the next time slot is the state of the system in the current time slot multiplied by the transition matrix $\mathbf{P}$. In the steady state, the following equation must hold: $\mathbf{v}=\mathbf{v} \cdot \mathbf{P}$. This, and the requirement that the sum of the state probabilities must be 1 , are sufficient to determine the steady state.

For the development of the Markov model, we start by defining the probability distribution of the number of blocked 
terminals $\mu$ as the state variable. Let $\mu$ be the average number of blocked terminals. We can then easily define our performance parameters. The channel traffic $T_{p}$ is the number of blocked terminals multiplied by the probability that they will transmit

$$
T_{p}=\mu \cdot p_{\mathrm{tr}}
$$

Given the number of terminals $N_{T}$ and the arrival probability $p$ of a packet at a terminal during a time slot, the throughput $S$ simply is

$$
S=p \cdot\left(N_{T}-\mu\right)
$$

(if, on average, $\mu$ terminals are blocked, then apparently the other $N_{T}-\mu$ terminals get their packets serviced at the same rate as they arrive).

The packet delay can be found by analyzing the possible paths a terminal can follow in the protocol. Looking back at Fig. 3, we see that there are three places where a packet can be delayed. The packet first experiences a delay when arriving at an idle terminal, on average half a time slot. The second type of delay occurs during the transmission of the packet, be it successful or not. Then, we have the delay waiting for the next time slot if a terminal decides not to transmit.

The delay model and its simplification are shown in Fig. 4. $p, q, s$, and $t$ represent the probability that a certain branch is chosen, $T$ is the duration of one time slot. $p$ is the transmission probability $p_{\mathrm{tr}}, q$ is its complement, $s=S / T_{p}$ is the probability that a transmission is successful, and $t$ is the complement of $s$. The probability that a delay of $0.5+n$ time slots is encountered is $p s(q+p t)^{n-1}$. The average delay $D$ is then

$$
D=\frac{1}{2}+\frac{T_{p}}{\left(p_{\text {str }} \cdot S\right)}
$$

where $p_{\text {str }}=p \cdot s$ is the probability of a successful transmission occurring during a time slot.

After establishing the performance parameters, we now focus on the transition matrix. In the network, we have a number of terminals at which packets arrive and a base station which can only service one packet per time slot at most. Examination of this network leads to five possible scenarios per time slot:

1) The network happens to be empty and zero or more arrivals occur.

2) The system state decreases by 1 because of a successful transmission and no new arrivals occur.

3) The system stays in the same state or moves up to

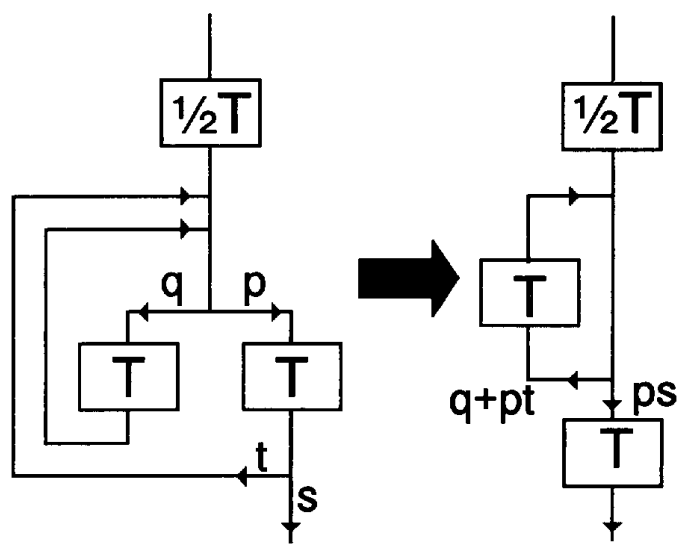

Fig. 4. Packet delay model.

any state but the highest due to multiple arrivals and a successful transmission.

4) The system jumps from the current state to the highest state because of multiple arrivals and no successful transmissions.

5) State decreases by more than 1 (probability 0 ).

Translating these scenarios into the transition probabilities, we get (4) at the bottom of the page, where $N_{T}$ is the number of terminals in the network, $i$ is the current state of the system, $j$ is the next state of the system, $p$ is the arrival probability per terminal, $q$ equals $1-p$, and $p_{\text {str }}$ is the probability that a successful transmission takes place during a time slot.

Equation (4) depends very much on $p_{\text {str }}$, which is a function of many variables including network topology and the distribution of blocked terminals. An expression for $p_{\text {str }}$ is determined in two steps. First, we look at the transmission process within a group of terminals sharing the same codes. Each blocked terminal has three possibilities: a successful transmission, an unsuccessful one, or no transmission at all. Once we have the success, collision, and silence probabilities for all groups, we can calculate $p_{\text {str }}$ by regarding each group as a new terminal in a supergroup transmitting to the base station.

\section{A. Terminal Probabilities}

We start with the probability $p_{\mathrm{ts}}$ that a transmitting terminal is successful. This depends on the transmission activity of other blocked terminals. Because the other blocked terminals usually are at different distances from the receiver belonging to the reference terminal, we have to take all possible combinations of transmitting and silent terminals into account. This

$$
p_{i, j}=\left\{\begin{array}{lll}
\left(\begin{array}{c}
N_{T} \\
j
\end{array}\right) p^{j} q^{\left(N_{T}-j\right)} & i=0 & \wedge 0 \leq j \leq N_{T} \\
p_{\mathrm{str}} q_{\left(N_{T}-i\right)}\left(\begin{array}{c}
N_{T}-i \\
p_{\text {str }}\left(\begin{array}{c}
N^{\prime}-i+1
\end{array}\right) p^{(j-i+1)} q^{\left(N_{T}-j-1\right)}
\end{array}\right. & 1 \leq i \leq N_{T} & \wedge j=i-1 \\
+\left(1-p_{\text {str }}\right)\left(\begin{array}{c}
N_{T}-i \\
j-i
\end{array}\right) p^{(j-i)} q^{\left(N_{T}-j\right)} & 1 \leq i \leq N_{T}-1 & \wedge 1 \leq j \leq i \\
\left(1-p_{\text {str }}\right) p^{N_{T}-i} & 1 \leq i \leq N_{T}-1 & \wedge j=N_{T} \\
0 & 2 \leq i \leq N_{T} & \wedge 0 \leq j \leq i-2
\end{array}\right.
$$


leads to a binomial distribution

$p_{\mathrm{ts}}\left(\vec{\mu}, p_{\mathrm{tr}}\right)=\sum_{i_{\mu}=0}^{1} \cdots \sum_{i_{1}=0}^{1} p_{\mathrm{tr}}^{x}\left(1-P_{\mathrm{tr}}\right)^{\mu-x}\left(1-p_{\mathrm{be}}\left(\vec{\mu}, p_{\mathrm{tr}}\right)\right)^{L_{p}}$

where $p_{\mathrm{tr}}$ is the probability that a blocked terminal transmits, $L_{p}$ is the packet length, $\vec{\mu}$ is a certain configuration of blocked terminals, and $x$ is the number of active terminals given by

$$
x=\sum_{v=1}^{\mu} i_{v}
$$

The last factor in brackets in (5) represents the packet success probability.

The probability $p_{\mathrm{tq}}$ that a blocked terminal is quiet is the complement of the probability that it transmits, so $p_{\mathrm{tq}}$ $=1-p_{\mathrm{tr}}$. A collision is the only other possibility, so the collision probability $p_{\mathrm{tc}}$ equals $1-p_{\mathrm{ts}}-p_{\mathrm{tq}}$.

\section{B. Group Probabilities}

The distributed receiver locks onto the signal of the first packet it receives and forwards it to the base station, whether it is a good packet or not. This capture process is modeled as an equiprobable selection of arriving packets. This means that if there are $i$ successful packets and $j$ unsuccessful packets, the probability that a good packet is sent to the base station is simply $i /(i+j)$.

Because there are three possible events (success, collision, and silence), we will obtain a trinomial distribution. The probability that a particular group is successful $p_{\mathrm{gs}}$ is

$$
p_{\mathrm{gs}}=\sum_{i=1}^{N_{t}} \sum_{j=0}^{N_{t}-i} \frac{i}{j+i} \cdot \frac{N_{t} !}{i ! j !\left(N_{t}-i-j\right) !} \cdot p_{\mathrm{ts}}^{i} p_{\mathrm{tc}}^{j} p_{\mathrm{tq}}^{N_{t}-i-j}
$$

where $N_{t}$ is the number of terminals per group.

The probability that a group is silent is simply the probability that all its terminals are silent

$$
p_{\mathrm{gs}}=\left(1-p_{\mathrm{tr}}\right)^{N_{t}}
$$

The probability that a collided packet is sent to the base station is $p_{\mathrm{gc}}=1-p_{\mathrm{gs}}-p_{\mathrm{gq}}$.

Unlike the terminal probabilities, the group probabilities will differ. Within the group, the terminal probabilities are the same because these terminals transmit to the same receiver. Because of the assumed perfect power control, all the signals from within the same group experience the same amount of interference. This is not so between groups in general.

\section{Successful Transmission Probability}

For the final group success probability, we have generalized (7) to

$$
\begin{aligned}
& p_{\text {str }}=\sum_{i_{n}=0}^{L_{n}} \sum_{j_{n}=0}^{L_{n}-i_{n}} \cdots \sum_{i_{1}=0}^{L_{1}} \sum_{j_{1}=0}^{L_{1}-i_{1}} \\
& \times\left(\frac{\sum_{k=1}^{n} i_{k}}{\sum_{k=1}^{n} i_{k}+\sum_{k=1}^{n} j_{k}} \cdot \prod_{v=1}^{n} \frac{L_{v}}{\left.i_{v} ! j_{v} !\left(L_{v}-i_{v}-j_{v}\right) !\right)}\right. \\
& \left.\times p_{\mathrm{gs}, \mathbf{v}}^{i_{v}} p_{\mathrm{gc}, \mathbf{v}}^{j_{v}} p_{\mathrm{gq}, \mathbf{v}}^{L_{v}-i_{v}-j_{v}}\right)
\end{aligned}
$$

where $n$ is the number of groups with blocked terminals, $L_{v}$ is the number of groups with blocked terminals with the same characteristics, and $\sum i_{k} /\left(\sum i_{k}+\sum j_{k}\right)=0$ whenever $\sum i_{k}=0$ and $\sum j_{k}=0$. A slight simplification is possible by realizing that all $i_{v}, j_{v}$, and $L_{v}$ are either 0 or 1 , so the fraction with the factorials may be replaced by 1 .

The probability of a successful transmission occurring depends on the bit error probability, for which an expression is derived in Section V, after presentation of the physical network model in the next section. In the calculation of the bit error probability for the Markov model, we assume that the blocked terminals are uniformly distributed over the network. As we will see in Section VI, this assumption is quite accurate.

\section{PHYSICAL MODEL}

\section{A. The Transmitter}

The transmitters first DPSK modulates the data signal and then spreads it with a 31-chip direct sequence Gold code. After modulation with a $1.7 \mathrm{GHz}$ carrier, the signal is transmitted. For our calculations, we assume an arbitrary data rate of 256 . $1024 \mathrm{~b} / \mathrm{s}(0.26 \mathrm{Mb} / \mathrm{s})$. The transmitter maintains perfect power control to ensure that the signals from within the same group arrive at the receiver with equal power. Since the terminals are not expected to move around during transmissions, power control is easily implemented.

\section{B. Channel Characteristics}

To gain full understanding of the hybrid protocol, we investigate two channel models. The first model assumes a noiseless nonfading channel in which the transmitted power is equally distributed over all paths. The second model assumes a noisy Rayleigh fading channel. For both models, extra cellular cochannel interference and a Line of Sight path are assumed to be absent. The number of resolvable paths $L$ is given by $\left\lfloor T_{m} / T_{c}\right\rfloor+1$ where $T_{m}$ is the RMS delay spread, $T_{c}$ is the chip duration, and $\lfloor x\rfloor$ denotes the largest integer smaller than or equal to $x$. An RMS delay spread of $100 \mathrm{~ns}$ coupled with a data rate of $0.26 \mathrm{Mb} / \mathrm{s}$ and 31 chip codes yields one resolvable path, considerably reducing calculation time compared with multipath environments.

As for the signal attenuation model, the far-field model was chosen because it is relatively easy to implement and easy to adapt to various environments. The path loss can be described as [9]

$$
S_{r}=\frac{g_{T} g_{R}}{\left(\frac{4 \pi f R}{c}\right)^{\alpha}} S_{t}
$$

where $S_{r}$ is the received power, $S_{t}$ is the transmitted power, $f$ is the transmission frequency, $R$ is the distance between transmitter and receiver, $g_{T}$ is the transmitter antenna gain, $g_{R}$ is the receiver antenna gain, $c$ is the speed of light, and $\alpha$ is the attenuation parameter. $\alpha$ can vary between 1.8 and 6 , depending on the morphology of the building. The former value corresponds with a transmitter and receiver in a hallway, where the hallway acts like a waveguide, the latter value corresponds to transmitters and receivers located in rooms perpendicular to a hallway with the doors closed. For ease of 
implementation $\alpha$ equals 2 in our calculations, corresponding to free-space propagation.

\section{The Receiver}

The receiver employs one antenna and no diversity except for the diversity inherent in Direct Sequence Spread Spectrum. The incoming signal is first translated to an intermediate frequency where it is despread using either a SAW filter or through digital sampling techniques. After that, the signal is DPSK demodulated and forwarded to the base station by wire. The receiver is noiseless as all thermal noise is accounted for in the channel model.

\section{Network Topology}

To evaluate several code sharing schemes, we place the receivers at a fixed distance from the base station and cluster the transmitters around the receivers, also at a fixed distance. The total number of terminals is chosen as a power of two. This way, we can double the number of codes and halve the group size several times. A 16-terminal network example is given in Fig. 5.

\section{BIT ERROR PROBABILITY}

This section presents the expressions for the bit error probability for two channel conditions, namely noiseless nonfading channels and Rayleigh fading channels with noise.

A. The basic expression for the bit error probability $p_{\text {be }}$ for DPSK with multipath propagation in $M$ time-invariant frequency-nonselective channels is given by Proakis [10]:

$$
p_{\text {be }}=\frac{1}{2^{(2 M-1)}} \operatorname{Exp}\left(-\gamma_{b}\right) \sum_{k=0}^{M-1}\left(\frac{\gamma_{b}^{k}}{k !} \sum_{n=0}^{M-1-k}\left(\begin{array}{c}
2 M-1 \\
n
\end{array}\right)\right)
$$

where $\gamma_{b}$ is the signal-to-noise ratio per bit. This formula is valid on the condition that the interfering signals can be treated as independent Gaussian noise. This is a reasonable assumption as it has been concluded in [11] using computer simulation that the approximation can be used to obtain the performance of a DS CDMA system analytically.

For the calculation of $\gamma_{b}$, we start with the signal $r_{u}(t)$, a distributed receiver belonging to reference user $u$ sees at its input

$$
\begin{aligned}
r_{u}(t)= & \sum_{k=1}^{K} \sum_{\ell=1}^{L} \sqrt{\frac{2 P_{k} s_{k u}^{2}}{L}} a_{k}\left(t-\tau_{k u \ell}\right) b_{k}\left(t-\tau_{k u \ell}\right) \\
& \times \operatorname{Cos}\left(\omega_{c} t+\theta_{k}+\phi_{k u \ell}\right)
\end{aligned}
$$

where $P_{k}$ is the power transmitted by user $k, s_{k u}$ is the amplitude gain over path $k$ - $u\left(0 \leq s_{k u} \leq 1\right), a_{k}$ is the $k$ th user code, $b_{k}$ is the $k$ th user databit, $\tau_{k u \ell}$ is the delay of path $\ell$ from user $k$ to reference user $u\left(0 \leq \tau_{k u \ell} \leq T_{b}\right), \phi_{k u \ell}$ is the phase shift of path $k u \ell\left(0 \leq \phi_{k u \ell} \leq 2 \pi\right), \theta_{k}$ is the initial carrier phase of user $k\left(0 \leq \theta_{k} \leq 2 \pi\right)$ and $L=$ number of resolvable paths. Without loss of generality, we assume that the initial carrier phase $\theta_{k}$ is absorbed by the path shift $\phi_{k u \ell}$.

Equation (12) can be written in a lowpass equivalent form

$$
r_{u}(t)=x_{u}(t) \operatorname{Cos}\left(\omega_{c} t\right)-y_{u}(t) \operatorname{Sin}\left(\omega_{c} t\right)
$$

with

$$
\begin{aligned}
x_{u}(t) & =\sum_{k=1}^{K} \sum_{\ell=1}^{L} A_{k u} a_{k}\left(t-\tau_{k u \ell}\right) b_{k}\left(t-\tau_{k u \ell}\right) \operatorname{Cos}\left(\phi_{k u \ell}\right) \\
y_{u}(t) & =\sum_{k=1}^{K} \sum_{\ell=1}^{L} A_{k u} a_{k}\left(t-\tau_{k u \ell}\right) b_{k}\left(t-\tau_{k u \ell}\right) \operatorname{Sin}\left(\phi_{k u \ell}\right) \\
A_{k u} & =\sqrt{\frac{2 P_{k} s_{k u}^{2}}{L}}
\end{aligned}
$$

So, the in-phase component is $x_{u}(t)$ and the quadrature component is $y_{u}(t)$.

Let us assume that our reference is path $h$ of user $u$. The in-phase and quadrature components after correlation at their sampling time $T_{b}$ can be described as

$$
\begin{aligned}
g_{x}\left(T_{b}\right)= & \sum_{k=1}^{K} \sum_{\ell=1}^{L} A_{k u} \operatorname{Cos}\left(\phi_{k u \ell}\right) \\
& \cdot\left[b_{k}^{-1} R_{u k}\left(\tau_{k u \ell}\right)+b_{k}^{0} \hat{R}_{u k}\left(\tau_{k u \ell}\right)\right] \\
g_{y}\left(T_{b}\right)= & \sum_{k=1}^{K} \sum_{\ell=1}^{L} A_{k u} \operatorname{Sin}\left(\phi_{k u \ell}\right) \\
& \cdot\left[b_{k}^{-1} R_{u k}\left(\tau_{k u \ell}\right)+b_{k}^{0} \hat{R}_{u k}\left(\tau_{k u \ell}\right)\right]
\end{aligned}
$$

in which $R_{u k}\left(\tau_{k u \ell}\right)$ and $\hat{R}_{u k}\left(\tau_{k u \ell}\right)$ are partial cross correlations

$$
\begin{aligned}
& R_{u k}(\tau)=\int_{0}^{\tau} a_{u}(t-\tau) a_{k}(t) d t \\
& \hat{R}_{u k}(\tau)=\int_{\tau}^{T_{b}} a_{u}(t-\tau) a_{k}(t) d t
\end{aligned}
$$

Assuming that the receiver associated with user $u$ is synchronized to a signal from user $u$, we have $\tau_{u u h}=0$ and $\phi_{u u h}=0$. The current sample value of the complex envelope of this lowpass equivalent signal $g_{x}(t)+j g_{y}(t)$ can be written as

$$
\begin{aligned}
Z_{0}= & A_{u u} T_{b} b_{u}^{0}+\sum_{k=1}^{K}\left(b_{k}^{-1} X_{k}+b_{k}^{0} \hat{X}_{k}\right) \\
& +j \sum_{k=1}^{K}\left(b_{k}^{-1} Y_{k}+b_{k}^{0} \hat{Y}_{k}\right)
\end{aligned}
$$

Similarly, we have for the complex envelope at the previous sampling instant

$$
\begin{array}{r}
Z_{-1}=A_{u u} T_{b} b_{u}^{-1}+\sum_{k=1}^{K}\left(b_{k}^{-2} X_{k}+b_{k}^{-1} \hat{X}_{k}\right) \\
+j \sum_{k=1}^{K}\left(b_{k}^{-2} Y_{k}+b_{k}^{-1} \hat{Y}_{k}\right)
\end{array}
$$




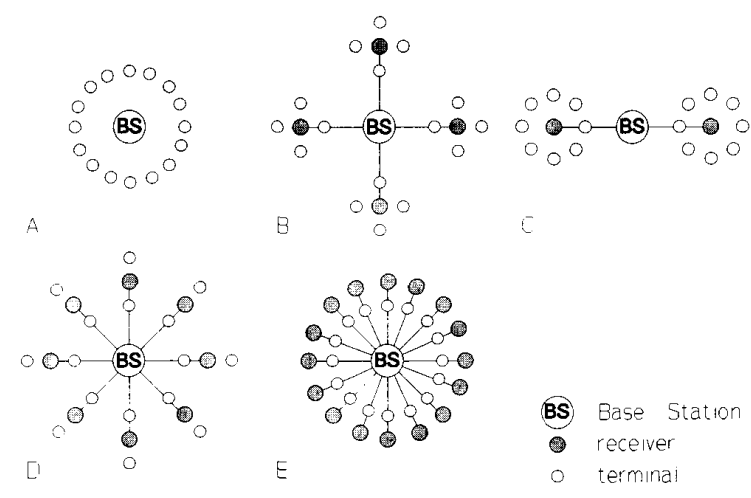

Fig. 5. Example of 16-terminal network topologies.

where

$$
\begin{aligned}
& X_{u}=\sum_{\substack{\ell=1 \\
\ell \neq h}}^{L} A_{u u} R_{u u}\left(\tau_{u u \ell}\right) \operatorname{Cos}\left(\phi_{u u \ell}\right) \\
& \hat{X}_{u}=\sum_{\substack{\ell=1 \\
\ell \neq h}}^{L} A_{u u} \hat{R}_{u u}\left(\tau_{u u \ell}\right) \operatorname{Cos}\left(\phi_{u u \ell}\right) \\
& Y_{u}=\sum_{\substack{\ell=1 \\
\ell \neq h}}^{L} A_{u u} R_{u u}\left(\tau_{u u \ell}\right) \operatorname{Sin}\left(\phi_{u u \ell}\right) \\
& \hat{Y}_{u}=\sum_{\substack{\ell=1 \\
\ell \neq h}}^{L} A_{u u} \hat{R}_{u u}\left(\tau_{u u \ell}\right) \operatorname{Sin}\left(\phi_{u u \ell}\right) \\
& X_{k}=\sum_{\ell=1}^{L} A_{k u} R_{u k}\left(\tau_{k u \ell}\right) \operatorname{Cos}\left(\phi_{k u \ell}\right) \\
& \hat{X}_{k}=\sum_{\ell=1}^{L} A_{k u} \hat{R}_{u k}\left(\tau_{k u \ell}\right) \operatorname{Cos}\left(\phi_{k u \ell}\right) \\
& Y_{k}=\sum_{\ell=1}^{L} A_{k u} R_{u k}\left(\tau_{k u \ell}\right) \operatorname{Sin}\left(\phi_{k u \ell}\right) \\
& \hat{Y}_{k}=\sum_{\ell=1}^{L} A_{k u} \hat{R}_{u k}\left(\tau_{k u \ell}\right) \operatorname{Sin}\left(\phi_{k u \ell}\right)
\end{aligned}
$$

All the terms of $Z_{0}$ and $Z_{-1}$, except the first one in (20) and (21), are regarded as interference. From now on, this interference signal is denoted as $Z_{i}$. The decision variable for the DPSK modulator is $\xi=\operatorname{Re}\left[Z_{0} \cdot Z_{-1}^{*}\right]=\operatorname{Re}\left[\left(A_{u u} T_{b} b_{u}^{0}+Z_{0, i}\right)\right.$. $\left.\left(A_{u u} T_{b} b_{u}^{-1}+Z_{-1, i}\right)^{*}\right]$ where $^{*}$ denotes complex conjugation.

The data bits are assumed to be equiprobable, and the probability of a bit error $p_{\text {be }}$ is given by [4]

$$
P_{\text {be }}=\operatorname{Pr}\left\{\xi<0 \mid b_{u}^{0} b_{u}^{-1}=1\right\}=\operatorname{Pr}\left\{\xi>0 \mid b_{u}^{0} b_{u}^{-1}=-1\right\}
$$

For the calculation of the interference power, we will assume that $b_{u}^{-1} \cdot b_{u}^{0}=1$ and that all other bit combinations such as $b_{k}^{-1} \cdot b_{i}^{0}(\neg(k=u \wedge i=u))$ are assumed to take on the values 1 and -1 with equal probability. This means that $E\left[b_{i}^{v} b_{j}^{w}\right]=$ $E\left[b_{i}^{v}\right] E\left[b_{j}^{w}\right]=0$ except for $i=j=u, v=-1$, and $w=0$. We used this property in calculating the expectation of the interference power over many samples. The interference power per bit equals $E\left[Z_{0, i} \cdot Z_{0, i}^{*}\right]$. By evaluation of this expression for the interference power, we developed an expression for the conditional interference power $N_{u} \mid\left(\left\{\tau_{k u \ell}\right\},\left\{\phi_{k u \ell}\right\}\right)$. In (20), the wanted signal amplitude is $A_{u u} T_{b} b_{u}^{0}$; therefore, the wanted signal power is $A_{u u}^{2} T_{b}^{2}$. This leads to

$$
\left.\gamma_{b}\right|_{\left(\left\{\tau_{k u \ell}\right\},\left\{\phi_{k u \ell}\right\}\right)}=\frac{A_{u u}^{2} T_{b}^{2}}{N_{u} \mid\left(\left\{\tau_{k u \ell}\right\},\left\{\phi_{k u \ell}\right\}\right)}
$$

The removal of the conditioning on $\tau_{k u \ell}$ and $\phi_{k u \ell}$ requires integration over these variables, which is not possible to do analytically. Instead, we used Monte Carlo Integration to obtain values for our Markov model. An important assumption is that the bit error probability remains the same throughout the packet. This is justified because the busy tone emitted by the base station forces the terminals to start within one bit duration of each other.

B. For the calculation of the bit error probability in noisy fading channels, we have used the formula given by Kavehrad and Ramamurthi for Rayleigh fading channels with first-order selection diversity [4]

$$
P_{\text {be }}=Q(a, b)-\frac{1}{2}\left(1+\frac{\mu_{12}}{\sqrt{\mu_{1} \mu_{2}}}\right) I_{0}(a b) \exp \left(-\frac{a^{2}+b^{2}}{2}\right)
$$

where

$$
\begin{aligned}
& a= \frac{m}{\sqrt{2}}\left|\frac{1}{\sqrt{\mu_{1}}}-\frac{1}{\sqrt{\mu_{2}}}\right| \quad b=\frac{m}{\sqrt{2}}\left(\frac{1}{\sqrt{\mu_{1}}}+\frac{1}{\sqrt{\mu_{2}}}\right) \\
& m= A_{u u} s_{u u, \max } T_{b} b_{1}^{0} \\
& \mu_{1}=E\left[\sum_{k=1}^{N_{t}} X_{k}^{2}+\hat{X}_{k}^{2}+Y_{k}^{2}+\hat{Y}_{k}^{2} \mid\left\{\tau_{k u \ell}\right\}, L\right] \\
&+ \\
&+E\left[X_{u} \hat{X}_{u}+Y_{u} \hat{Y}_{u} \mid\left\{\tau_{k u \ell}\right\}, L\right]+2 \sigma_{n}^{2} \\
& \mu_{2}=E\left[\sum_{k=1}^{N_{t}} X_{k}^{2}+\hat{X}_{k}^{2}+Y_{k}^{2}+\hat{Y}_{k}^{2} \mid\left\{\tau_{k u \ell}\right\}, L\right]+2 \sigma_{n}^{2} \\
& \mu_{12}=E\left[\sum_{k=1}^{N_{t}}\left(X_{k} \hat{X}_{k}+Y_{k} \hat{Y}_{k}\right)+X_{u}^{2}+Y_{u}^{2} \mid\left\{\tau_{k u \ell}\right\}, L\right]
\end{aligned}
$$

and $\sigma_{n}^{2}$ is the thermal noise power.

\section{RESULTS}

For the results in this section, we have used the following parameters: delay spread $100 \mathrm{~ns}$, a fixed terminal- to-receiver distance, and a fixed receiver-to-base station distance for networks using the hybrid protocol, random terminal positioning for CDMA-only networks, free space signal attenuation $(\alpha=2), 31$-chip Gold codes, a data rate $0.26 \mathrm{Mb} / \mathrm{s}$, an SNR of $20 \mathrm{~dB}$ in noisy channels, and a packet length of 64 bits unless stated otherwise. It is worth noting that the delay spread in buildings varies from 50 to $250 \mathrm{~ns}$ [12], [13].

We begin with a comparison of the Markov model and simulation results for eight terminal networks without retransmission delay, with noiseless nonfading channels (Fig. 6). Terminal-to-receiver distance is $5 \mathrm{~m}$, receiver-to-base station distance is $20 \mathrm{~m}$. Both Markov model and simulation results agree quite well. Because the performance was virtually the same for all network geometries (code reuse schemes), we show only the results of using one code for different transmis- 


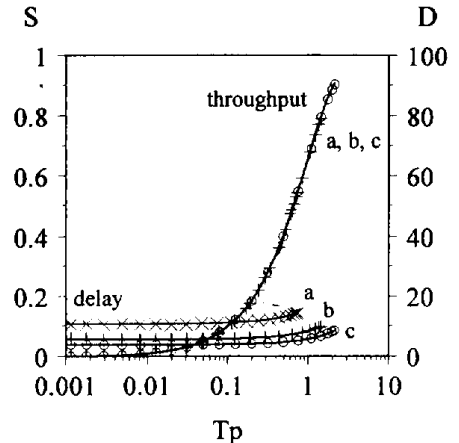

Fig. 6. Comparison between Markov model (solid lines) and simulation results (markers), of an eight-terminal network using one 31-chip code for a noiseless nonfading channel. ac performance for $p_{\mathrm{tr}}=0.1(x), 0.2(+)$ $0.3(\mathrm{O})$.

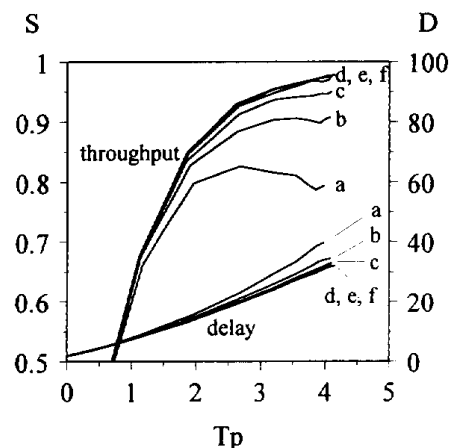

Fig. 7. Effect of coding on 32-terminal network performance with a fixed transmission probability of 0.7 for a noisy fading channel. a.f: $1,2,4,8,16$, and 32 codes. Code length $=31$

sion probabilities. In this case, code sharing does not incur a performance penalty. The throughput curves are the same for all three values of the transmission probability $p_{\mathrm{tr}}$, for both simulation and calculation. The only difference is that, for higher $p_{\mathrm{tr}}$, the maximum throughput is higher. This result can be explained by the fact that the network is not heavily loaded for these values of $p_{\mathrm{tr}}$ and eight users. The delays experienced are due to the randomization of transmission attempts. Most of the transmissions succeed at the first attempt. Consequently, the performance is quite good.

The following results are for noisy Rayleigh fading channels. The transmission probability is 0.7 , and the geometrically distributed retransmission delay is on average 4 and 8 time slots for 16 and 32 terminal networks, respectively. For the hybrid protocol, the terminal-to-receiver distance is $5 \mathrm{~m}$ and the receiver-to-base station distance is $30 \mathrm{~m}$. The ripples in the simulation curves are caused by statistical variance of the simulation results.

Fig. 7 presents the effects of code sharing for a 32-terminal network with distributed receivers. The influence of the number of transmission codes used is only visible at high traffic levels. Using 4 codes would suffice for this network.

Fig. 8 shows the influence of multipath propagation on 32 terminal network performance by changing the code sequence length and data rate, for both the hybrid protocol with only

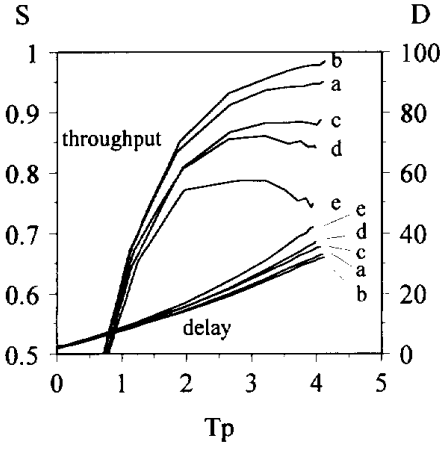

Fig. 8. Effect of multipath propagation on network performance for a noisy fading channel: a) hybrid with 31-chip codes, b) hybrid with 127-chip codes, c) hybrid with 31-chip codes and high bit rate, d) CDMA with 31-chip codes, e) CDMA with 31-chip codes and high bit rate.

four codes and conventional CDMA with 32 codes. Plot $a$ represents the performance of a four-code 31 -chip hybrid protocol. Plot $b$ illustrates the effect of increasing the code length to 127 , and plot $c$ the effect of quadrupling the bit rate to 1.04 $\mathrm{Mb} / \mathrm{s}$. In both cases, the number of resolvable paths increases from 1 to 4 , but as could be expected case $b$ results in a better performance and case $c$ in a poorer performance. Plots $d$ and $e$ illustrate the degradation in performance for standard CDMA when the bit rate is increased. Under equal circumstances, the hybrid protocol performs better than CDMA.

Finally, to demonstrate the robustness of the hybrid protocol we compare network performance when the number of terminals is increased from 16 to 32 for CDMA and the hybrid protocol. For both hybrid protocol networks, 4 codes are used. Fig. 9 shows the simulation results for high traffic loads. The CDMA performance shows signs of degradation when the number of terminals is doubled, whereas the hybrid protocol continues to provide a high throughput with less delay than CDMA. This result suggests that a higher number of users can be supported by the hybrid protocol than by CDMA when using the same bandwidth.

\section{CONCLUSION}

The throughput and delay have been evaluated for the proposed hybrid CDMA/ISMA protocol considering Direct Sequence Spread Spectrum CDMA and p-persistent ISMA with DPSK modulation scheme for data communications in ideal and Rayleigh fading indoor environments.

A Markov model of the proposed hybrid CDMA/ISMA protocol is developed. The results of this model are compared with the simulation results for eight terminals. A close agreement has been observed between analytical and simulation results. Therefore, it is recommended to evaluate the performance of the hybrid CDMA/ISMA protocol using computer simulation for large numbers of terminals, because it gives results fast. Computational results further show that for the 32-terminal networks, 4 codes yield quite good performance. In a normal CDMA system, to achieve similar performance one needs 32 different codes.

In a Rayleigh fading channel, the computational results show that the performance is enhanced by increasing the code 
$\mathrm{S}$

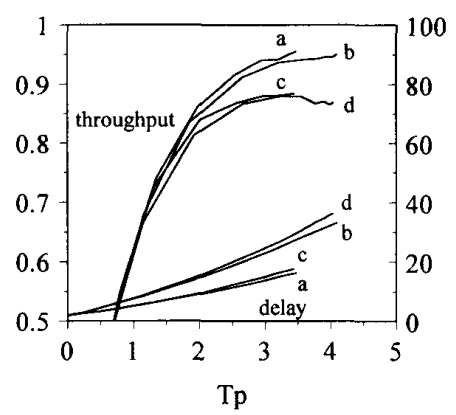

Fig. 9. Effect on performance of increase in network size for CDMA and hybrid protocols for a noisy fading channel: a) 16 terminal hybrid network, b) 32 terminal hybrid network, c) 16 terminal CDMA network, d) 32 terminal CDMA network.

sequence length, but decreases when the data rate is increased. It is worth to mention that the performance is reduced only when the increase of the data rate results in more resolvable paths.

Code sharing proves to be a feasible technique to support many users using a limited number of short transmission codes. Further simulation results indicate that the hybrid protocol can support a higher number of users more successfully than ordinary CDMA.

\section{REFERENCES}

[1] R. Prasad, "Performance analysis of mobile packet radio networks in real channels with inhibit sense multiple access," Proc. Inst. Elec. Eng. $I$, vol. 138 , pp. $485-464,1991$.

[2] R. Prasad and C. Y. Liu, "Throughput analysis of some mobile packet radio protocols in Rician fading channels," Proc. Inst. Elec. Eng. I, vol. 139, no. 3, pp. 297-302, June 1992.

[3] R. Prasad and C. A. F. J. Wijffels, "Performance analysis of a slotted CDMA system for indoor wireless communication using a Markov chain model," in Proc. IEEE Global Telecommun. Conf., Dec. 2-5, 1991, pp. 1953-1957.

[4] M. Kavehrad and B. Ramamurthi, “ Direct-sequence spread spectrum with DPSK modulation and diversity for indoor wireless communications," IEEE Trans. Commun., vol. COM-35, no. 2, pp. 224-236, Feb.1987,

[5] H. S. Misser, C. A. F. J. Wijffels, and R. Prasad, "Throughput analysis of CDMA with DPSK modulation and diversity in indoor Rician fading radio channels," Elect. Lett., vol. 27, no. 7, pp. 601-602, Mar. 1991.

[6] D. Raychaudhuri, "Performance analysis of random access packetswitched code division multiple access systems," IEEE Trans.Commun., vol. COM-29, no. 6, pp. 895-901, June 1981.

[7] J. M. Musser and J. N. Daigle, "Throughput analysis of an asynchronous code division multiple access (CDMA) system," in Proc. Int. Cong. Commun. (ICC), 1982, pp. 2.F.2.1-7.

[8] M. B. Pursley, "Performance evaluation for phase coded spread spectrum multiple access communication-Part I: System analysis," IEEE Trans. Commun., vol. COM-25, no. 8, pp. 795-798, Aug. 1977.

[9] A. B. Carlson, Communication Systems. New York: McGraw Hill, 1988.

[10] J. G. Proakis, Digital Communications. McGraw Hill, 1989.

[11] H. S. Misser, A. Kegel, and R. Prasad, "Monte Carlo simulation of direct sequence spread spectrum for indoor radio communication in a Rician fading channel," Proc. Inst. Elec. Eng. I, vol. 139, no. 6, pp. 620-624, Dec. 1992.

[12] D. M. J. Devasirvatham, "Time delay spread and signal level measurements of $850 \mathrm{MHz}$ radio waves in building environments," IEEE Trans. Antenn. and Prop., vol. AP-34, no. 11, pp. 1300-1305, Nov. 1986.

[13] R. J. C Bultitude, "A comparison of indoor radio propagation characteristics at $910 \mathrm{MHz}$ and $1.75 \mathrm{MHz}$," IEEE J. Select. Areas Commun., vol. SAC-7, no. 1, pp. 20-30, Jan. 1989.

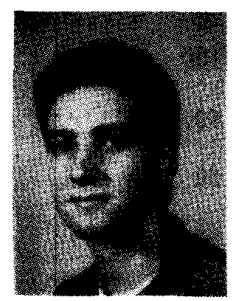

Huub van Roosmalen was born on September 25 , 1968. He received the Engineering degree from The Delft University of Technology, Delft, The Netherlands in August 1992.

Besides his work on spread spectrum communications, he has participated in research on the micromechanical properties of glass-fiber reinforced polyester at the Faculty of Mechanical Engineering. Currently, he holds a position with the Dutch Ministry of Defense.

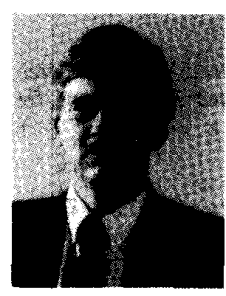

Jos A. M. Nijhof was born in Rijswijk, The Netherlands, in 1945. He received the M.Sc. degree in electrical engineering from the Delft University of Technology in 1971 .

After graduation, he joined the TU Delft Centre for Traffic and Transportation Engineering as a Research Coordinator. Since 1975, he has been with the Telecommunications and Traffic Control Systems Group of Delft University of Technology, where he is now an Associate Professor in data communications systems and networks. He contributes regularly to courses and seminars on data communications, network architectures, mobile communications, and performance analysis of telecommunication systems. His research interests are protocols and access techniques for broadband and wireless communications networks.

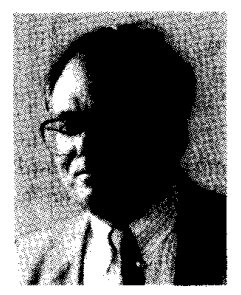

Ramjee Prasad (M'88-SM'90) was born in Babhnaur (Gaya), Bihar, India on July 1, 1946. He received the B.Sc. (Eng.) degree from the Bihar Institute of Technology, Sindri, India, and the M.Sc. (Eng.) and Ph.D. degrees from the Birla Institute of Technology (BIT), Ranchi, India in 1968, 1970, and 1979 , respectively.

He joined BIT as Senior Research Fellow in 1970 and became Associate Professor in 1980. During 1983-1988, he was with the University of Dar es Salaam (UDSM), Tanzania, where he became Professor of Telecommunications in the Department of Electrical Engineering in 1986. Since February 1988, he has been with the Telecommunications and Traffic Control Systems Group, Delft University of Technology, The Netherlands, where he is actively involved in the area of mobile, indoor, and personal radio communications. While he was with BIT, he supervised many research projects in the area of microwave and plasma engineering. At UDSM, he was responsible for the collaborative project "Satellite Communications for Rural Zones" with the Eindhoven University of Technology in The Netherlands. He has published over 150 technical papers. His current research interests involve packet communications, adaptive equalizers, spread-spectrum CDMA systems, and multimedia communications. He has served as a member of advisory and program committees of several IEEE international conferences. He has also presented tutorials on mobile and indoor radio communications at various universities, technical institutions, and IEEE conferences. He is also a member of a working group of European cooperation in the field of scientific and technical research (COST-231 project) dealing with "Evolution of Land Mobile Radio (including Personal) Communications" as an expert for the Netherlands. He is listed in the U.S. Who's Who in the World. He was Organizer and Interim Chairman of the IEEE Vehicular Technology/Communications Society Joint Chapter, Benelux Section. Now he is the Elected Chairman of the Joint Chapter. He is also founder of the IEEE Symposium on Communications and Vehicular Technology (SCVT) in the Benelux and he was the Symposium Chairman of SCVT'93. He is one of the Editors-in-Chief of a new journal on wireless personal communications and also a member of the Editorial Board of other international journals including the IEEE Communications Magazine. He is the Technical Program Chairman of the PIMRC'94 International Symposium to be held in The Hague, The Netherlands during September 19-23, 1994 and also of the Third Communication Theory Mini-Conference in conjunction with GLOBECOM'94 to be held in San Francisco, CA during November 27-30, 1994. He is a Fellow of IEE, a Fellow of the Institution of Electronics \& Telecommunication Engineers, and a member of the New York Academy of Sciences. 